Proc. Estonian Acad. Sci. Phys. Math., 2006, 55, 2, 75-95

\title{
Robust pole assignment via stable polytopes of reflection vectors
}

\author{
Ülo Nurges \\ Institute of Cybernetics at Tallinn University of Technology, Akadeemia tee 21, 12618 Tallinn, \\ Estonia; nurges@ioc.ee
}

Received 2 March 2006

\begin{abstract}
A robust version of the output controller design for discrete-time systems is introduced. Instead of a single stable point a stable polytope (or simplex) is preselected in the coefficient space of closed-loop characteristic polynomials. A constructive procedure for generating stable simplexes is given starting from the unit hypercube of reflection coefficients of monic polynomials. This procedure is quite straightforward, because for a special family of polynomials the linear cover of so-called reflection vectors is stable. The root placement of reflection vectors is studied. If a stable target simplex is preselected, then the robust output controller design task is solved by the quadratic programming approach.
\end{abstract}

Key words: pole placement, discrete-time systems, robust stability, quadratic programming.

\section{INTRODUCTION}

The modal control or pole placement method is a common approach for designing closed-loop controllers in order to meet desired control specifications. The objective of assigning closed-loop poles is often replaced by assigning a characteristic polynomial, because this polynomial plays a central role in the stability analysis of linear control systems and polynomial coefficients are simply (affinely) related to controller and plant parameters. By state feedback arbitrary pole assignment is possible if the system is controllable. For output feedback the problem of classical pole placement is in general unsolvable by fixed-order controller. That is why much effort has been devoted to the regional pole placement problem, the objective of which is to place closed-loop poles in a suitable region of the complex plane $\left[{ }^{1-4}\right]$.

Another practical issue is that of model uncertainty. If the model uncertainty is relatively small, then it is possible to use sensitivity-based methods. If the model 
uncertainty is large, some robust formulation of the problem is needed, such as the multimodel $\left[{ }^{5,6}\right]$, polytopic model $\left[{ }^{7,8}\right]$ or LMI approach $\left[{ }^{9,10}\right]$.

The main disadvantage of the parametric methods is the well-known fact that the stability domain in the space of polynomial coefficients is nonconvex in general. That is why several convex approximations of the stability region, such as ellipsoids $\left[{ }^{11,12}\right]$, hyperrectangles $\left[{ }^{13-15}\right]$, and polytopes $\left[{ }^{7,8,16}\right]$, are well known and widely used in robust control.

In this paper a polytopic approach is developed. First, instead of a fixed single point in the coefficient space of closed-loop characteristic polynomials, a stable simplex (or polytope) is preselected. A family of polynomials is defined, which generates a Schur stable linear cover of so-called reflection vectors.

Second, we consider a polytopic plant model, i.e., the set of possible plant parameters is defined as a convex polytope. This kind of modelling allows the determination of properties that are common to all elements in the set from the analysis of its vertices only. Thus the complexity of computations is determined by the number of vertices of the polytope.

If a stable simplex is preselected, then the robust output controller design task for the polytopic plant model can be solved by the quadratic programming approach.

The paper is organized as follows. In Section 2 the problem of fixed-order robust output control with a preselected simplex is stated and solved by the quadratic programming approach. The next three sections consider the development of the method for preselection of a stable simplex via reflection coefficients of monic polynomials. In particular, the stability region is studied via reflection coefficients in Section 3. In Section 4 the stable polytope of so-called reflection vectors is introduced for a special family of stable polynomials. In Section 5 the root placement of reflection vectors is studied in order to give suggestions for preselection of a target simplex. The last section is devoted to the robust output control of uncertain (polytopic) plants.

\section{FIXED-ORDER POLE ASSIGNMENT}

Assume that a plant with parametric uncertainties is given. Our goal is to design an output controller of a fixed order so that the closed-loop poles are robustly assigned in a specific region.

For simplicity, let us first consider the problem of output controller design for a SISO plant with fixed parameters. Let the plant transfer function $G(z)$ of dynamic order $m$ be given:

$$
G(z)=\frac{g(z)}{f(z)}=\frac{g_{m-1} z^{m-1}+\cdots+g_{1} z+g_{0}}{z^{m}+\cdots+f_{1} z+f_{0}} .
$$

We are looking for a controller $C(z)$ of dynamic order $l$ with the transfer function 


$$
C(z)=\frac{q(z)}{p(z)}=\frac{q_{l} z^{l}+\cdots+q_{1} z+q_{0}}{z^{l}+\cdots+p_{1} z+p_{0}} .
$$

It means that the closed-loop characteristic polynomial

$$
a(z)=f(z) p(z)+g(z) q(z)
$$

is of degree $n=m+l$.

It is known $\left[{ }^{1}\right]$ that when $l=m-1$, the above problem has a solution for arbitrary $a(z)$ whenever the plant has no common pole-zero pairs. In general, for $l<m-1$ exact attainment of the desired polynomial is impossible. Here we suggest the following approach.

Let us relax the requirement of attaining the desired polynomial $a(z)$ exactly and enlarge the target to a simplex $\mathcal{S}$ in the polynomial coefficient space containing the point representing the desired closed-loop characteristic polynomial. Without any restrictions we can assume that $f_{m}=p_{l}=1$ and deal in the following with monic polynomials $a(z)$.

Let us now introduce a stability measure $\rho$ in accordance with the simplex $\mathcal{S}$ :

$$
\rho=c^{T} c,
$$

where

$$
c=S^{-1} a
$$

and $S$ is the $(m+l+1) \times(m+l+1)$ matrix of vertices of the target simplex $\mathcal{S}$. Obviously, for monic polynomials

$$
\sum_{i=1}^{n+1} c_{i}=1,
$$

where $n=m+l$. If all coefficients $c_{i}>0, i=1, \ldots, n+1$, then the point $a$ is placed inside the simplex $\mathcal{S}$.

It is easy to see that the minimum of $\rho$ is obtained by

$$
c_{1}=c_{2}=\ldots=c_{n+1}=\frac{1}{n+1} .
$$

Then the point $a$ is placed in the centre of the simplex $\mathcal{S}$.

Now we can formulate the following problem of controller design: find a controller $C(z)$ such that the stability measure $\rho$ is minimal. In other words, we are looking for a controller which places the closed-loop characteristic polynomial $a(z)$ as close as possible to the centre of the target simplex $\mathcal{S}$.

In matrix form we have

$$
a=G x,
$$


where $G$ is the plant Sylvester matrix

$$
G=\left[\begin{array}{cccccccc}
f_{0} & 0 & \ldots & 0 & g_{0} & 0 & \cdots & 0 \\
f_{1} & f_{0} & \ldots & 0 & g_{1} & g_{0} & \cdots & 0 \\
\cdot & \cdot & \cdot & \cdot & \cdot & \cdot & \cdot & \cdot \\
f_{m-1} & f_{m-2} & \ldots & f_{m-l-1} & g_{m-1} & g_{m-2} & \cdots & g_{m-l-1} \\
1 & f_{m-1} & \cdots & f_{m-l} & 0 & g_{m-1} & \cdots & g_{m-l} \\
0 & 1 & \cdot & \cdot & . & . & . & \cdot \\
0 & 0 & \cdots & f_{m-1} & 0 & 0 & \cdots & g_{m-1} \\
0 & 0 & \cdots & 1 & 0 & 0 & \cdots & 0
\end{array}\right]
$$

of dimensions $(m+l+1) \times(2 l+2)$ and $x$ is the $(2 l+2)$-vector of controller parameters $x=\left[p_{0}, \ldots, p_{l-1}, 1, q_{0}, \ldots, q_{l}\right]^{T}$.

Due to relations (1) and (2) the above controller design problem is equivalent to the quadratic programming problem: find $x$ such that the minimum

$$
J_{1}=\min _{x} x^{T} G^{T}\left(S S^{T}\right)^{-1} G x
$$

is obtained subject to the linear restrictions

$$
S^{-1} G x>0 .
$$

Here the restriction follows from the positivity requirement of coefficients $c_{i}$, $i=1, \ldots, n$. For monic polynomials the requirement $\sum c_{i}=1$ is automatically satisfied.

Sometimes $J_{1}$, which is a kind of distance to the centre of the target simplex $\mathcal{S}$, is not the best minimization criterion because certain restrictions must be considered by choosing the vertices of the target simplex $\mathcal{S}$ (see Section 4). Then it is better to use another criterion $J_{2}$, which measures the distance to a fixed Schur polynomial $e(z)$ serving as a "nominal" closed-loop polynomial:

$$
J_{2}=(a-e)^{T}(a-e)=(G x-e)^{T}(G x-e) .
$$

In general, we can use a weighted combination of the criteria $J_{1}$ and $J_{2}$,

$$
J=(1-\alpha) J_{1}+\alpha J_{2}, \quad 0 \leq \alpha \leq 1,
$$

and solve the quadratic programming task

$$
\begin{gathered}
J=\min _{x}\left\{x^{T} G^{T}\left[(1-\alpha)\left(S S^{T}\right)^{-1}+\alpha I_{n+1}\right] G x-2 \alpha e^{T} G x\right\}, \\
S^{-1} G x>0 .
\end{gathered}
$$

Let us consider now the problem of fixed-order output controller design, where the plant is subject to parameter uncertainty. We represent this by supposing that the 
given plant transfer function coefficients $f_{0}, \ldots, f_{m-1}$ and $g_{0}, \ldots, g_{m-1}$ are placed in a polytope $\mathcal{W}$ with vertices $d^{j}=\left[f_{0}^{j}, \ldots, f_{m-1}^{j}, g_{0}^{j}, \ldots, g_{m-1}^{j}\right], j=1, \ldots, M$ :

$$
\mathcal{W}=\operatorname{conv}\left\{d^{j}, j=1, \ldots, M\right\} .
$$

Because the relations (2) are linear in plant parameters, we can claim that for an arbitrary fixed controller $x$ the vector $a$ of closed-loop characteristic polynomial coefficients is placed in a polytope $\mathcal{A}$ with vertices $a^{1}, \ldots, a^{M}$ :

$$
\mathcal{A}=\operatorname{conv}\left\{a^{j}, j=1, \ldots, M\right\},
$$

where

$$
a^{j}=D^{j} x
$$

and $D^{j}$ is an $(m+l+1) \times(2 r+2)$ Sylvester matrix composed by the vertex plant $d^{j}$ as in the case of exact model (2).

The problem of robust controller design can be formulated as follows: find a controller $x$ such that all vertices $a^{j}, j=1, \ldots, M$, are placed inside a stable target simplex $\mathcal{S}$.

This problem can be solved by a quadratic programming task: find $x$ which minimizes

$J=\min _{x}\left\{x^{T} \tilde{D}^{T}\left[(1-\alpha)\left(I_{M} \otimes\left(S^{T}\right)^{-1}\right)\left(I_{M} \otimes S^{-1}\right)+\alpha I_{(n+1) M}\right] \tilde{D} x-2 \alpha e^{T} \tilde{D} x\right\}$

by linear restrictions

$$
S^{-1} D^{j} x>0, \quad j=1, \ldots, M .
$$

Here $I_{M}$ is the unit matrix of order $M, \otimes$ denotes the Kronecker product, and $\tilde{D}^{T}=\left[D_{1}^{T}, \ldots, D_{M}^{T}\right]$.

In the following three sections a novel method for the convex inside approximation of the stability region will be developed via so-called reflection coefficients of polynomials. This method can be used to find a stable target simplex in order to solve the robust output pole assignment problem (4), (5).

\section{STABILITY REGION VIA REFLECTION COEFFICIENTS}

Polynomials are usually defined in terms of their coefficients or their roots. They can also be characterized by their reflection coefficients using Schur-Cohn type recursion $\left[{ }^{17}\right]$.

Let $a_{n}(z)$ be a monic polynomial of degree $n$ with real coefficients $a_{i} \in \mathcal{R}$, $i=0, \ldots, n$,

$$
a(z)=z^{n}+\ldots+a_{1} z+a_{0} .
$$


The reciprocal polynomial $a_{n}^{*}(z)$ of $a_{n}(z)$ is defined by $\left[{ }^{17}\right]$ :

$$
a_{n}^{*}(z)=a_{0} z^{n}+\ldots+a_{n-1} z+1 .
$$

The reflection coefficients $k_{i}, i=1, \ldots, n$, can be obtained from $a_{n}(z)$ by using backward Levinson's recursion $\left[{ }^{18}\right]$

$$
z a_{i-1}(z)=\frac{1}{1-\left|k_{i}^{2}\right|}\left[a_{i}(z)-k_{i} a *_{i}(z)\right]
$$

where $k_{i}=-a_{i, 0}$ and $a_{i, 0}$ denotes the last coefficient of an $i$-degree polynomial $a_{i}(z)$. From (6) the forward recursion can be obtained:

$$
a_{i}(z)=z a_{i-1}(z)+k_{i} a_{i-1}^{*}(z) .
$$

From (6) and (7) the coefficient expressions of $a_{i-1}(z)$ and $a_{i}(z)$ are given, respectively, by

$$
a_{i-1}(z)=\frac{1}{1-\left|k_{i}^{2}\right|}\left[\sum_{j=0}^{i-1}\left(a_{i, j+1}-k_{i} a_{i, i-j-1}\right) z^{j}\right]
$$

and

$$
a_{i}(z)=\sum_{j=0}^{i}\left(a_{i-1, j-1}+k_{i} a_{i-1, i-j-1}\right) z^{j} .
$$

The reflection coefficients $k_{i}$ are also known in the literature as SchurSzegö parameters $\left[{ }^{17}\right]$, partial correlation (PARCOR) coefficients $\left[{ }^{19}\right]$ or $k$-parameters $\left[{ }^{20}\right]$. They have been used efficiently in many applications in signal processing $\left[{ }^{20}\right]$ and system identification $\left[{ }^{19}\right]$.

A complete characterization and classification of polynomials using reflection coefficients instead of roots (zeros) of polynomials is given in $\left[{ }^{17}\right]$. The main advantage of using reflection coefficients is the fact that the transformation from reflection coefficients to polynomial coefficients is very simple. Indeed, according to (7) or (9), polynomial coefficients $a_{i}$ depend multilinearly on reflection coefficients $k_{i}$. If the coefficients are real $a_{i} \in \mathcal{R}$, then also the reflection coefficients are real $k_{i} \in \mathcal{R}$.

The transformation from reflection coefficients $k_{i}, i=1, \ldots, n$, to polynomial coefficients $a_{i}, i=0, \ldots, n-1$, can be presented as

$$
\begin{gathered}
a_{i}=a_{n-i}^{(n)}, \\
a_{i}^{(i)}=-k_{i}, \\
a_{j}^{(i)}=a_{j}^{(i-1)}-k_{i} a_{i-j}^{(i-1)}, \quad i=1, \ldots, n ; \quad j=1, \ldots, i-1
\end{gathered}
$$


or in the matrix form

$$
\begin{array}{r}
a=R(k) a^{(t)}, \quad t=1, \ldots, n-1, \\
a^{(t)}=\left[\begin{array}{c}
0^{T} \\
R_{t}\left(k_{t}\right)
\end{array}\right] a^{(t-1),}
\end{array}
$$

where

$$
\begin{gathered}
a=\left[a_{0}, \ldots, a_{n-1}, 1\right]^{T}, \\
a^{(t)}=\left[0, a_{t}^{(t)}, \ldots, a_{1}^{(t)}, 1\right]^{T}, \\
a^{(0)}=[0,1]^{T}, \\
R(k)=R_{n}\left(k_{n}\right)\left[\begin{array}{c}
0^{T} \\
R_{n-1}\left(k_{n-1}\right)
\end{array}\right] \ldots\left[\begin{array}{c}
0^{T} \\
R_{t}\left(k_{t}\right)
\end{array}\right], \\
R_{j}\left(k_{j}\right)=I_{j+1}-k_{j} E_{j+1},
\end{gathered}
$$

$I_{n}$ is an $n \times n$ unit matrix, $E_{n}$ is a unit Hankel matrix $E_{n}=\left[\begin{array}{ccc}0 & \ldots & 1 \\ . & \cdot & . \\ 1 & \ldots & 0\end{array}\right]$, and $0^{T}$ is a row vector of zeros.

A linear discrete-time dynamic system is stable if its characteristic polynomial is Schur stable, i.e., if all its poles lie inside the unit circle. The stability criterion via a reflection coefficient is as follows $\left[{ }^{17}\right]$.

Lemma 1. A polynomial $a(z)$ has all its roots inside the unit disk if and only if $\left|k_{i}\right|<1, i=1, \ldots, n$.

A polynomial $a(z)$ lies on the stability boundary if some $k_{i}= \pm 1, i=1, \ldots, n$. For monic Schur polynomials there is a one-to-one correspondence between the vectors $a=\left(a_{0}, \ldots, a_{n-1}\right)^{T}$ and $k=\left(k_{1}, \ldots, k_{n}\right)^{T}$.

The stability region in the reflection coefficient space is simply the $n$-dimensional unit hypercube $\mathcal{K}=\left\{k_{i} \in(-1,1), i=1, \ldots, n\right\}$. We can find the stability region in the polynomial coefficient space starting from the unit hypercube $\mathcal{K}$. The mapping (10) is one-to-one for monic Schur polynomials. However, for the stability boundary this transformation is not one-to-one.

The next theorem describes the stability boundary in the polynomial coefficient space starting from the stability hypercube $\mathcal{K}$ of reflection coefficients.

Theorem 1. The stability boundary in the reflection coefficient space is composed of $2 n$ faces $k_{i}= \pm 1, i=1, \ldots, n$, of the hypercube $\mathcal{K}$. These faces will be transformed by mapping (10) to the following boundary surfaces in the polynomial coefficient space:

1) for $k_{n}=1$ and $n$ odd an $(n+1) / 2$-dimensional hyperplane

$$
\left\{\begin{array}{l}
a_{0}=-1, \\
a_{j}=-a_{n-j}, \quad j=1, \ldots,(n-1) / 2
\end{array}\right.
$$


2) for $k_{n}=1$ and $n$ even an $n / 2$-dimensional hyperplane

$$
\left\{\begin{array}{l}
a_{0}=-1, \\
a_{n / 2}=0, \\
a_{j}=-a_{n-j}, \quad j=1, \ldots, n / 2-1 ;
\end{array}\right.
$$

3) for $k_{n}=-1$ and $n$ even an $(n+1) / 2($ or $n / 2+1)$-dimensional hyperplane

$$
\left\{\begin{array}{l}
a_{0}=1, \\
a_{j}=a_{n-j},
\end{array}\right.
$$

where $j=1, \ldots,(n-1) / 2$ when $n$ is odd or $j=1, \ldots, n / 2-1$;

4) for $k_{i}=1$ and $i$ even an $(n-2)$-dimensional hyperplane

$$
\left\{\begin{array}{c}
a_{0}+a_{1}+\ldots+a_{n-1}+1=0 \\
a_{0}+\ldots+a_{n-i / 2-1}+a_{n-i / 2+1} \\
+\ldots+a_{n-1}+1=0
\end{array}\right.
$$

5) for $k_{i}=1$ and $i$ odd an $(n-1)$-dimensional hyperplane

$$
a_{0}+a_{1}+\ldots+a_{n-1}+1=0
$$

6) for $k_{i}=-1$ and $i$ odd an $(n-1)$-dimensional hyperplane

$$
a_{0}-a_{1}+\ldots+(-1)^{n-1} a_{n-1}+1=0 ;
$$

7) for $k_{i}=-1$, $i$ even, and $i<n$ an $n$-dimensional surface

$$
a=R_{n}\left(k_{n}\right) \ldots\left[\begin{array}{c}
0^{T} \\
R_{i}(-1)
\end{array}\right] \cdots\left[\begin{array}{c}
0^{T} \\
R_{1}\left(k_{1}\right)
\end{array}\right]\left[\begin{array}{l}
0 \\
1
\end{array}\right] .
$$

Proof. The proof is quite straightforward and follows immediately from the transformation (11) from reflection coefficients to polynomial coefficients.

For $k_{n}=1$ we obtain from (11)

$$
a=\left[\begin{array}{ccccc}
1 & 0 & \ldots & 0 & -1 \\
0 & 1 & \ldots & -1 & 0 \\
. & . & . & . & . \\
0 & -1 & \ldots & 1 & 0 \\
-1 & 0 & \ldots & 0 & 1
\end{array}\right] a^{(n-1)},
$$

i.e., (12) holds.

If now $n$ is even, then $a_{n / 2}=0$ and (13) holds. Similarly we obtain (14) for $k_{n}=-1$. 
Now let us consider the matrix $R_{i}\left(k_{i}\right)$ for $i$ even:

$$
R_{i}\left(k_{i}\right)=\left[\begin{array}{ccccc}
1 & 0 & \ldots & 0 & -k_{i} \\
\cdot & \cdot & \cdot & \cdot & \cdot \\
0 & \ldots & 1-k_{i} & \ldots & 0 \\
\cdot & \cdot & \cdot & \cdot & \cdot \\
-k_{i} & 0 & \ldots & 0 & 1
\end{array}\right]
$$

Let us denote

$$
\begin{gathered}
s_{t}=[1, \ldots, 1] a^{(t)}, \\
\bar{s}_{t}=\left[(-1)^{t+1}, \ldots,-1,1\right] a^{(t)}, \\
s_{0}=\bar{s}_{0}=1 .
\end{gathered}
$$

Then we obtain from (11)

$$
\begin{gathered}
s_{t}=\left(1-k_{t}\right) s_{t-1}, \\
\bar{s}_{t}=\left[1+(-1)^{t-1} k_{t}\right] \bar{s}_{t-1} .
\end{gathered}
$$

Obviously,

$$
\begin{gathered}
s_{n}=[1, \ldots, 1] a, \\
\bar{s}_{n}=\left[(-1)^{n}, \ldots,-1,1\right] a .
\end{gathered}
$$

If $k_{i}=1$, then the $(i / 2)$ th column of the matrix $R_{i}\left(k_{i}\right)$ is a 0 -vector. It means

$$
s_{n}=a_{0}+\ldots+a_{n-i / 2-1}+a_{n-i / 2+1}+\ldots+a_{n-1}+1 .
$$

For $k_{i}=1, i \in\{1, \ldots, n\}$, we have $s_{n}=0$, i.e., (15) holds.

From (19) we obtain

$$
s_{n}=\left(1-k_{1}\right)\left(1-k_{2}\right) \ldots\left(1-k_{n}\right)
$$

and hence $s_{n}=0$ if $k_{i}=1, i \in\{1, \ldots, n\}$, i.e., (16) holds.

Similarly, from (20) we obtain

$$
\bar{s}_{n}=\left(1+k_{1}\right)\left(1-k_{2}\right) \ldots\left(1+(-1)^{n-1} k_{n}\right)
$$

and $\bar{s}_{n}=0$ if $k_{i}=-1, i$ odd, i.e., (17) holds.

Equation (18) follows immediately from (11) for $t=1$ and $a^{(0)}=[0,1]^{T}$. 


\section{STABLE POLYTOPE OF REFLECTION VECTORS}

In this section we show that for a family of polynomials the linear cover of so-called reflection vectors is Schur stable.

The next lemma forms the basis for the following development. It follows immediately from the multilinear transformation (10) between reflection coefficients and polynomial coefficients $\left[{ }^{21}\right]$.

Lemma 2. Through an arbitrary stable point $a=\left(a_{0}, a_{1}, \ldots, a_{n-1}\right)$ with reflection coefficients $k_{i}^{a} \in(-1,1), i=1, \ldots, n$, one can draw $n$ stable line segments

$$
\mathcal{V}^{i}(a)=\operatorname{conv}\left\{a \mid k_{i}^{a}= \pm 1\right\},
$$

where $\operatorname{conv}\left\{a \mid k_{i}^{a}= \pm 1\right\}$ denotes the convex hull obtained by varying the reflection coefficient $k_{i}^{a}$ between -1 and 1 .

The endpoints of the line segments $\mathcal{V}^{i}(a)$ are called reflection vectors of the polynomial $a(z)$.

Definition 1. The reflection vectors of a Schur stable monic polynomial a $(z)$ are defined as the points on the stability boundary in the polynomial coefficient space generated by changing a single reflection coefficient $k_{i}$ of the polynomial a $(z)$.

Let us denote the positive reflection vectors of the polynomial $a(z)$ as

$$
v_{i}^{+}(a)=\left(a \mid k_{i}=1\right), i=1, \ldots, n,
$$

and the negative reflection vectors of the polynomial $a(z)$ as

$$
v_{i}^{-}(a)=\left(a \mid k_{i}=-1\right), i=1, \ldots, n .
$$

The following assertions hold:

1) every Schur polynomial has $2 n$ reflection vectors $v_{i}^{+}(a)$ and $v_{i}^{-}(a), i=1, \ldots, n$;

2) all the reflection vectors lie on the stability boundary $\left(k_{i}^{v}= \pm 1\right)$;

3 ) the line segments between reflection vectors $v_{i}^{+}(a)$ and $v_{i}^{-}(a)$ are Schur stable.

In the following theorem we define a family of stable polynomials such that the polytope generated by the reflection vectors of these polynomials is stable.

Theorem 2. Let $k_{1}^{a} \in(-1,1), k_{n}^{a} \in(-1,1)$, and $k_{2}^{a}=\ldots=k_{n-1}^{a}=0$. Then the inner points of the polytope $\mathcal{V}(a)$, generated by the reflection vectors of the point a,

$$
\mathcal{V}(a)=\operatorname{conv}\left\{v_{i}^{ \pm}(a), \quad i=1, \ldots, n\right\}
$$

are Schur stable. 
Proof. We use the well-known edge theorem $\left[{ }^{22}\right]$ to prove this theorem. It means we have to show that all the edges

$$
\mathcal{E}_{i j}(a)=\operatorname{conv}\left\{v_{i}^{ \pm}(a), v_{j}^{ \pm}(a), i, j=1, \ldots, n\right\}
$$

of the polytope $\mathcal{V}(a)$ are stable.

First, let us mention that by Lemma 2 all the line segments between the reflection vectors $v_{i}^{+}(a)$ and $v_{i}^{-}(a)$

$$
\mathcal{E}_{i i}(a)=\operatorname{conv}\left\{v_{i}^{+}(a), v_{i}^{-}(a), i=1, \ldots, n\right\}
$$

are stable.

Second, let us consider the edges

$$
\mathcal{E}_{i j}(a)=\operatorname{conv}\left\{v_{i}^{ \pm}(a), v_{j}^{ \pm}(a) ; i, j=1, \ldots, n\right\} .
$$

Let us start from the polytope $\mathcal{V}(b)$ generated by reflection vectors of a monic polynomial $b(z)$ with reflection coefficients $k_{1} \in(-1,1), k_{2}=\ldots=k_{n}=0$,

$$
\mathcal{V}(b)=\operatorname{conv}\left\{v_{i}^{ \pm}(b), i=1, \ldots, n\right\} .
$$

It is shown in $\left[{ }^{23}\right]$ that the inner points of the polytope $\mathcal{V}(b)$ are Schur stable.

Now we can find an arbitrary point $\bar{a}$ of the edge $\mathcal{E}_{i j}(a), \bar{a} \in \mathcal{E}_{i j}(a)$ by the linear transformation (11) with $R_{n}\left(k_{n} \in(-1,1)\right)$ from a point $\bar{b}$ of the line segment $\mathcal{E}_{i j}(b), \bar{b} \in \mathcal{E}_{i j}(b)$ with $k_{n}^{b}=0, k_{i}^{b}=k_{i}^{a}, i=1, \ldots, n-1$ :

$$
\bar{a}=R_{n}\left(k_{n}\right) \bar{b} .
$$

The line segments

$$
\mathcal{E}_{i j}(b)=\operatorname{conv}\left\{v_{i}^{ \pm}(b), v_{j}^{ \pm}(b) ; i, j=1, \ldots, n-1\right\}
$$

are stable (or on the stability boundary) as the edges of the polytope $\mathcal{V}(b)$.

The line segments $\mathcal{E}_{i n}(a)$ can be obtained by (11) from the line segments

$$
\mathcal{E}_{i}(b)=\operatorname{conv}\left\{v_{i}^{ \pm}(b), b\right\},
$$

which are stable as the line segments between the point $b$ and the vertices of the polytope $\mathcal{V}(b)$.

According to (11), the transformation from the points $\bar{b}$ to the points $\bar{a}$ is linear with $R_{n}\left(k_{n} \in(-1,1)\right)$ and does not change any of the reflection coefficients $k_{i}, \quad i=1, \ldots, n-1$. So the edges $\mathcal{E}_{i j}(a)$ will be stable if $k_{i}^{b} \in(-1,1)$, $i=1, \ldots, n-1$, and $k_{n}^{a} \in(-1,1)$.

Because all the edges of the polytope $\mathcal{V}(a)$ are stable (or on the stability boundary), the inner points of the polytope $\mathcal{V}(a)$ are stable. 
Let us compare the proposed method of stability region approximation by polytopes of reflection vectors with the method of inner ellipsoidal approximation $\left[{ }^{12}\right]$ via the volumes of polytopes of reflection vectors and stable ellipsoids. For different values of degree $n$, we computed the volumes of stable polytopes of reflection vectors for initial polynomials $a(z)$ with $k_{2}^{a}=\ldots=k_{n}^{a}=0$, and (I) $k_{1}^{a}=0$, and (II) $k_{1}^{a}=+1$. As indicated in Section 6 these polytopes of relection vectors with $0 \leq k_{1}^{a} \leq 1$ and $k_{2}^{a}=\ldots=k_{n}^{a}=0$ are most suitable for robust controller design. In the first row of Table 1 the volumes of stability ellipsoids $\left[{ }^{12}\right]$, in the second row the volumes of stability diamonds with $k_{1}^{a}=\ldots=k_{n}^{a}=0\left[{ }^{23}\right]$, and in the third row the volumes of stability polytopes with $k_{1}^{a}=+1, k_{2}^{a}=\ldots=k_{n}^{a}=0$ are given. In Table 1 we can see that volumes of ellipsoids are always slightly greater than volumes of diamonds, but the volumes of polytopes are considerably greater than volumes of ellipsoids for $n>2$.

Example 1. Let $n=2$. Then the stability region in the polynomial coefficient space $a=\left(a_{1}, a_{0}\right)$ is the triangle FGH (Fig. 1). Let us find some stability region inside approximations according to the above theorem.

Let us start from the polynomial $a(z)=z^{2}-0.75 z+0.5$ (point $\mathbf{A}$ in Fig. 1) with reflection coefficients $k_{1}(a)=0.5, k_{2}(a)=-0.5$. According to Lemma 2, we can draw two line segments through the point $\mathbf{A}$. By varying the first reflection coefficient $k_{1},-1<k_{1}<1$, we get the line segment $\mathbf{F B}$ and by varying the second

Table 1. Volumes of stable coefficient space domains for discrete-time polynomials

\begin{tabular}{l|c|c|c|c}
\hline & $n=2$ & $n=3$ & $n=4$ & $n=5$ \\
\hline Ellipsoid & 2.2479 & 1.4790 & 0.7770 & 0.3176 \\
Diamond $k_{1}=0$ & 2.0 & 1.3333 & 0.6667 & 0.2667 \\
Polytope $k_{1}=1$ & 2.0 & 2.9814 & 1.3333 & 0.5333
\end{tabular}

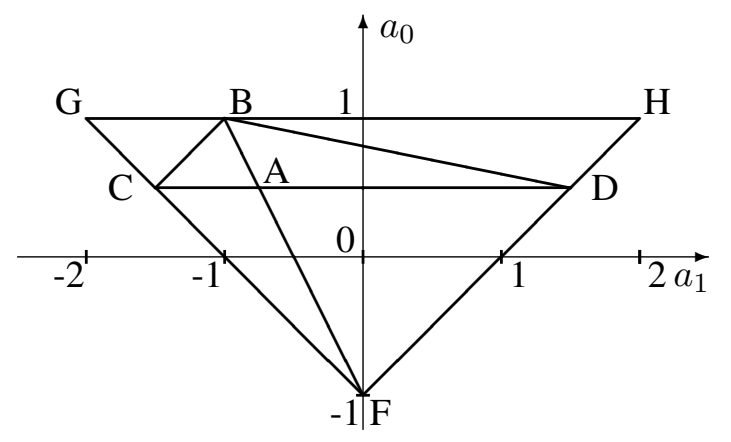

Fig. 1. Stable polytope of reflection vectors $(n=2)$. 
reflection coefficient $k_{2},-1<k_{2}<1$, we get the line segment CD. By definition the second-order polynomial $a(z)$ has four reflection vectors:

$$
\begin{aligned}
& v_{1}^{+}(a)=\left[\begin{array}{rr}
0.5 & -1.5
\end{array}\right], \\
& v_{1}^{-}(a)=\left[\begin{array}{rr}
0.5 & 1.5
\end{array}\right], \\
& v_{2}^{+}(a)=\left[\begin{array}{cr}
-1 & 0
\end{array}\right], \\
& v_{2}^{-}(a)=\left[\begin{array}{cr}
1 & -1
\end{array}\right] .
\end{aligned}
$$

According to Theorem 2, the inner points of the polytope BCDF of reflection vectors are stable.

\section{ROOTS OF REFLECTION VECTORS}

In this section we study the root placement of reflection vectors. It is useful for selecting a stable target simplex in order to solve the robust output control problem $\left[{ }^{24}\right]$.

By definition, at least one of the roots of a reflection vector $v_{i}(a)$ (i.e. a root of a polynomial $\left.v_{i}(z)=\left[1 z \ldots z^{n}\right]\left[\begin{array}{c}v_{i}(a) \\ 1\end{array}\right]\right)$ must lie on the unit circle. The following theorem states that the number of unit circle roots is determined by the number $i$ of the reflection vector $v_{i}(a)$ and the character of the roots (real or complex) is determined by the sign of the boundary reflection coefficient $\left(k_{i}^{v}= \pm 1\right)$.

Theorem 3. The reflection vectors $v_{i}^{+}(a)$ and $v_{i}^{-}(a), i=1, \ldots, n$, of a monic Schur polynomial $a(z)$ have $i$ roots $r_{j}, j=1, . ., i$, on the stability boundary. The numbers of real and complex roots are determined by the sign and the parity of the reflection vector as follows:

1) the positive reflection vector $v_{i}^{+}(a)$ has

- for $i$ even $r_{1}=1$,

$$
\begin{aligned}
& r_{2}=-1, \\
& \text { and }(i-2) / 2 \text { pairs }
\end{aligned}
$$

of complex roots on the unit circle,

- for $i$ odd $r_{1}=1$,

$$
\text { and }(i-1) / 2 \text { pairs }
$$

of complex roots on the unit circle,

2) the negative reflection vector $v_{i}^{-}(a)$ has

- for $i$ even $i / 2$ pairs

of complex roots on the unit circle,

- for $i$ odd $r_{1}=-1$,

$$
\text { and }(i-1) / 2 \text { pairs }
$$

of complex roots on the unit circle.

To prove this theorem, we need some preliminary lemmas. 
Lemma 3. The reflection vectors $v_{i}^{ \pm}(a)$ of an nth-order monic Schur polynomial $a(z)$ have $n$ roots on the unit circle.

Proof. First, let us mention that, according to Theorem 1, the reflection vectors $v_{n}^{ \pm}(a)$ have symmetric coefficient values. For $v_{n}^{+}(a)$ we have from (12) and (13):

$$
a_{j}=-a_{n-j}, \quad j=1, \ldots, n / 2(\operatorname{or}(n-1) / 2)
$$

and for $v_{n}^{-}(a)$ from (14)

$$
a_{j}=a_{n-j}, \quad j=1, \ldots, n / 2(\operatorname{or}(n-1) / 2) .
$$

Second, for monic polynomials with symmetric coefficient values it is well known that the number of roots inside the unit circle equals the number of roots outside the unit circle. But all the reflection vectors of Schur polynomials are by definition placed on the stability boundary. Hence, $v_{n}^{ \pm}(a)$ has no root outside the unit circle, and so all the $n$ roots of it are placed on the unit circle.

Lemma 4. Let us consider monic Schur polynomials $a(z)$ and $b(z)$ with reflection coefficients $k_{j}^{a}=k_{j}^{b}, j=1, \ldots, i-1, k_{j}^{a} \neq k_{j}^{b}, j>i$. Then the reflection vectors $v_{i}^{ \pm}(a)$ and $v_{i}^{ \pm}(b)$ have the same $i$ roots on the unit circle.

Proof. Let us start from an auxiliary $i$ th-order polynomial $\bar{a}^{i}(z)$ with reflection coefficients $k_{j}^{\bar{a}}=k_{j}^{a}=k_{j}^{b}, j=1, \ldots, i$. According to Lemma 3 , the polynomial $\bar{a}^{i}(z)$ has $i$ roots on the unit circle and its coefficients have symmetric values. For $k_{j}^{a}=k_{j}^{b}=-1$ we have

$$
\bar{a}^{i}(z)=z^{i}+a_{1} z^{i-1}+a_{2} z^{i-2}+\ldots+a_{2} z^{2}+a_{1} z+1
$$

and for $k_{j}^{a}=k_{j}^{b}=1$,

$$
\bar{a}^{i}(z)=z^{i}+a_{1} z^{i-1}+a_{2} z^{i-2}+\ldots-a_{2} z^{2}-a_{1} z-1 .
$$

Our aim is to show that the polynomial $\bar{a}^{i}(z)$ is a common divisor of both $a(z)$ and $b(z)$. It can be easily done by increasing the order of polynomials $a(z)$ and $b(z)$ by transformation (11) and taking into account the symmetric coefficient values of $\bar{a}^{i}(z)$.

For example, let $k_{i}^{a}=-1, i$ even and $\operatorname{deg}(a)=i+1$. Then

$$
a=\left[\begin{array}{ccccc}
1 & 0 & \ldots & 0 & -k_{i+1} \\
. & \cdot & \cdot & \cdot & \cdot \\
0 & \ldots & 1-k_{i+1} & \ldots & 0 \\
-k_{i+1} & 0 & \ldots & 0 & \dot{1}
\end{array}\right]\left[\begin{array}{c}
0 \\
1 \\
1-a_{1} k_{i} \\
a_{1}-a_{2} k_{i} \\
\vdots \\
a_{2}-a_{1} k_{i} \\
a_{1}-k_{i} \\
1
\end{array}\right]
$$


and

$$
a(z)=\left(z-k_{i}\right) \bar{a}^{i}(z) .
$$

For $\operatorname{deg}(a)=i+2$ we obtain

$$
a(z)=\left[z^{2}+k_{i+1}\left(k_{i+2}-1\right) z-k_{i+2}\right] \bar{a}^{i}(z) .
$$

In a similar way we can find for $\operatorname{arbitrary} \operatorname{deg}(a)$ and $\left|k_{j}\right|<1, j=i+1, \ldots, \operatorname{deg}(a)$,

$$
a(z)=a^{n-i}(z) \bar{a}^{i}(z)
$$

i.e., $\bar{a}^{i}(z)$ is a common divisor for all $a(z)$ (and also for $b(z)$ ) with $\operatorname{deg}(a)>i$.

Lemma 5. Let a monic polynomial a $(z)$ of order $n$ have a real root of multiplicity $i$ on the Schur stability boundary $r_{1}=r_{2}=\ldots=r_{i},\left|r_{i}\right|=1$ and all the other roots be placed inside the unit circle $\left|r_{j}\right|<1, j=i+1, \ldots, n$. Then the first $i$ reflection coefficients of $a(z)$ are placed on the stability boundary $\left|k_{j}\right|=1, j=1, \ldots, i$, and all the other reflection coefficients are inside the unit hypercube $\left|k_{j}\right|<1$, $j=i+1, \ldots, n$.

Proof. By Lemma 4 the $i$ th reflection coefficient of $a(z)$ must be of modulus equal to one, $\left|k_{i}\right|=1$, and $\left|k_{j}\right|<1, j=i+1, \ldots, n$, because $a(z)$ has only $i$ roots on the stability boundary. Now let us rewrite $a(z)$ as follows:

$$
a(z)=a^{n-i}(z) \bar{a}^{i}(z)=a^{n-i}(z)(z \pm 1)^{i} .
$$

Because $\bar{a}^{i}(z)$ has symmetric coefficient values, we can claim that the first $i$ reflection coefficients of $a(z)$ are determined by $\bar{a}^{i}(z)$, i.e., $k_{j}^{\bar{a}}=k_{j}^{a}, j=1, \ldots, i$. By formulas (10) we can easily check that for $\left|k_{j}^{\bar{a}}\right|=1, j=1, \ldots, i$, we have $\bar{a}^{i}(z)=(z \pm 1)^{i}$.

Proof of Theorem 3. The assertion that a reflection vector $v_{i}^{ \pm}(a)$ has $i$ roots on the unit circle follows immediately from Lemmas 3 and 4.

Now the question is: which of the reflection vectors has a real root on the stability boundary and of what sign? By Theorem 1 the hyperplanes (16) and (17) are the real root boundaries for $r=1$ and $r=-1$, respectively, and (15) is the real root boundary for both $r= \pm 1$. Because all the reflection vectors of a Schur polynomial have by definition only one reflection coefficient on the stability boundary, by Lemma 5 they have no real multiple roots of modulus equal to one. Hence, all the rest of the stability boundary roots must be complex roots on the unit circle. 


\section{ROBUST OUTPUT CONTROLLER DESIGN}

Let us return now to the problem of robust output control. We are looking for a robust output controller such that the closed-loop characteristic polynomial is placed in a stable polytope (linear cover) of reflection vectors. It means that the following problems have to be solved:

1) choice of an initial polynomial $a(z)$ for generating the polytope $\mathcal{V}(a)$,

2) choice of $n+1$ most suitable vertices of the polytope $\mathcal{V}(a)$ to build a target simplex $\mathcal{S}$,

3) choice of a target polynomial $e(z)$ according to (4).

In the following some "thumb rules" are given for choosing a stable target simplex $\mathcal{S}$.

According to Theorem 2, the initial polynomial $a(z)$ belongs to the family of polynomials with $k_{1}^{a} \in(-1,1), k_{n}^{a} \in(-1,1)$, and $k_{2}^{a}=\ldots=k_{n-1}^{a}=0$. Let us study the root placement of such polynomials.

1) Let $k_{1}^{a} \in(-1,1)$ and $k_{2}^{a}=\ldots=k_{n}^{a}=0$. Then $a(z)=z^{n}-k_{1} z^{n-1}$ and $r_{1}=k_{1}, r_{2}=\ldots=r_{n}=0$.

2) Let $k_{n}^{a} \in(-1,1)$ and $k_{1}^{a}=\ldots=k_{n-1}^{a}=0$. Then $a(z)=z^{n}-k_{n}$ and the roots of $a(z)$ are placed symmetrically against the origin whereas $\max \left|r_{i}\right|>k_{n}$.

The roots of a polynomial with a sufficient stability margin must be placed in a circle with radius $\beta<1$ and centre $\gamma$ on the positive real axis so that $\beta+\gamma<1\left[{ }^{5}\right]$. Thus, a reasonable choice of an initial polynomial is the following: $0<k_{1}^{a}<1$, $\left|k_{n}^{a}\right|<<k_{1}, k_{2}^{a}=\ldots=k_{n-1}^{a}=0$.

To choose $n+1$ vertices of the target simplex $\mathcal{S}$, we make use of Theorem 3 . It is well known that the poles with the positive real part are preferred to those with the negative real part $\left[{ }^{5}\right]$. Thus, according to Theorem 3 , the positive reflection vectors $v_{i}^{+}(a)$ with $i$ odd and negative reflection vectors $v_{i}^{-}(a)$ with $i$ even are chosen. It gives us $n$ vertices. The $(n+1)$ th vertex of the target simplex $\mathcal{S}$ is chosen as the mean of the remaining reflection vectors.

The target polynomial $e(z)$ of order $n$ can be, in principle, chosen freely. Nevertheless, it is reasonable to choose it inside the stable polytope of reflection vectors $\mathcal{V}(a)$. A quite common choice is $e(z)=a(z)$.

For higher-order polynomials the volume of a target simplex $\mathcal{S}$ is considerably less than the volume of the polytope of reflection vectors $\mathcal{V}$. That is why the above quadratic programming method with a preselected target simplex $\mathcal{S}$ works only if uncertainties are sufficiently small. Otherwise it is reasonable to use some search procedure to find a robust controller such that the polytope of closed-loop characteristic polynomials is placed inside the stable polytope of reflection vectors $\mathcal{V}(a)$.

Let us consider now a very simple example to explain the main ideas of robust fixed-order output controller design via the preselection of reflection vector polytopes and the quadratic programming task (4),(5). 
Example 2. Let us have the second-order $m=2$ uncertain plant with the transfer function

$$
G(z)=\frac{z+(0.6 \pm 0.1)}{z^{2}-(0.8 \pm 0.2) z-0.4}
$$

and a proportional $l=0$ output controller

$$
C(z)=q .
$$

Because $l<m-1$, we cannot choose an arbitrary closed-loop characteristic polynomial. Indeed,

$$
a(z)=z^{2}-[(0.8 \pm 0.2)+q] z+[(0.6 \pm 0.1) q-0.4]
$$

or, in matrix form,

$$
a=G x=\left[\begin{array}{cc}
f_{0} & g_{0} \\
f_{1} & g_{1} \\
1 & 0
\end{array}\right]\left[\begin{array}{l}
1 \\
q
\end{array}\right] .
$$

Let us choose, according to the above "thumb rules", the reflection coefficients $k_{1}^{a}=0.2$ and $k_{2}^{a}=0$. Then the generating polynomial $a(z)=z^{2}-0.2 z$ has four reflection vectors (points $\mathbf{C}, \mathbf{D}, \mathbf{F}, \mathbf{A}$ in Fig. 2, respectively):

$$
\begin{aligned}
& v_{1}^{+}(a)=\left[\begin{array}{cr}
-1 & 0
\end{array}\right], \\
& v_{1}^{-}(a)=\left[\begin{array}{cr}
1 & 0
\end{array}\right], \\
& v_{2}^{+}(a)=\left[\begin{array}{cr}
0 & -1
\end{array}\right], \\
& v_{2}^{-}(a)=\left[\begin{array}{ll}
-0.4 & 1
\end{array}\right] .
\end{aligned}
$$

The three vertices of the target simplex $\mathcal{S}=\operatorname{conv}(\mathbf{A}, \mathbf{C}, \mathbf{E})$ are chosen as $\mathbf{C}=v_{1}^{+}(a), \mathbf{A}=v_{2}^{-}(a)$, and $\mathbf{E}=0.5\left(v_{1}^{-}(a)+v_{2}^{+}(a)\right)$. So the matrix $S$ of vertex polynomial coefficients is

$$
S=\left[\begin{array}{ccc}
0 & 1 & -0.5 \\
-1 & -0.4 & 0.5 \\
1 & 1 & 1
\end{array}\right]
$$

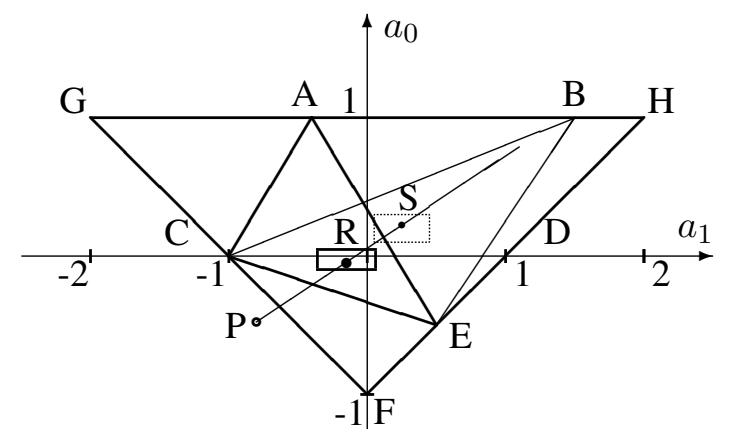

Fig. 2. Target simplexes for robust controller design. 
Let us now solve the output controller design task for the nominal plant with $g_{0}=0.6$ and $f_{1}=-0.8$ (point $\mathbf{P}$ ) via quadratic programming. Taking $\alpha=0$ in the optimization criterion $J(4)$, we obtain the controller gain

$$
q^{1}=0.5405
$$

and the closed-loop characteristic polynomial (point $\mathbf{R}$ )

$$
a^{1}(z)=z^{2}-0.2595 z-0.0757 .
$$

For polytopic plant with $g_{0}=0.6 \pm 0.1$ and $f_{1}=-0.8 \pm 0.2$ we obtain the controller gain

$$
q^{2}=0.6417
$$

and the vertices of the rectangle of closed-loop characteristic polynomials (solid rectangle around $\mathbf{R}$ )

$$
\begin{aligned}
& a^{21}(z)=z^{2}+0.0417 z+0.0492 \\
& a^{22}(z)=z^{2}+0.0417 z-0.0792 \\
& a^{23}(z)=z^{2}-0.3583 z+0.0492 \\
& a^{24}(z)=z^{2}-0.3583 z-0.0792
\end{aligned}
$$

To illustrate the effect of the choice of reflection coefficients of the generating polynomial $a(z)$, we have solved the same task with $k_{1}^{a}=-0.8$. Then the target simplex $\mathcal{S}$ is the triangle BCE. The optimal controller gain for the nominal plant is $q^{3}=1.0659$, which gives the closed-loop characteristic polynomial (point $\mathbf{S}$ )

$$
a^{3}=z^{2}+0.2659 z+0.2396
$$

For the polytopic plant we obtain

$$
\begin{gathered}
q^{4}=1.0141 \\
a^{41}(z)=z^{2}+0.4141 z+0.3099 \\
a^{42}(z)=z^{2}+0.4141 z+0.107 \\
a^{43}(z)=z^{2} 0.0141 z+0.3099 \\
a^{44}(z)=z^{2} 0.0141 z+0.107 .
\end{gathered}
$$

The value of the optimization criterion $J$ indicates in some sense (the less the better) the stability margin of the closed-loop polytope against the target simplex, $\frac{1}{n+1}<J_{1}<n+1$. For $k_{1}^{a}=0.2$ we have $J(0.2)=0.8272$ and for $k_{1}^{a}=-0.8$, $J(-0.8)=0.7659$. Thus it seems that $k_{1}^{a}=-0.8$ gives a slightly better result, but we have to take into account also the root placement of target simplexes. In general, for $0<k_{1}^{a}<1$ the root placement is better than for $-1<k_{1}^{a}<0$.

Indeed, the closed-loop polytope has better root placement for $k_{1}^{a}=0.2$ (solid rectangle) than for $k_{1}^{a}=-0.8$ (dotted rectangle). 
Example 3. Let us consider now the problem of designing a robust controller for the approximate model of a PUMA 762 robotic disk grinding process $\left[{ }^{9}\right]$. From the results of identification and because of the nonlinearity of the robot, the coefficients of the numerator of the plant transfer function change for different positions of the robot arm

$$
\begin{aligned}
& (0.0257 \pm 0.0005) z^{3}-(0.0764 \pm 0.01528) z^{2} \\
& G(z)=\frac{-(0.1619 \pm 0.03238) z-(0.1688 \pm 0.03376)}{z^{4}-1.9140 z^{3}+1.7790 z^{2}-1.0265 z+0.2508} \text {. }
\end{aligned}
$$

Let us fix the controller order $l=3$. Then $n=m+l=7$. By choosing the initial generating polynomial $a(z)=z^{7}-0.5 z^{6}$, i.e., $k_{1}^{a}=0.5, k_{2}^{a}=\ldots=k_{7}^{a}=0$, we obtain by the above approach for the robust controller

$$
C(z)=\frac{0.4168 z^{3}-0.7210 z^{2}-0.0638 z-0.0866}{z^{3}+1.4162 z^{2}+0.9267 z+0.2805} .
$$

Because of the great uncertainty level (up to $20 \%$ around the nominal value of the parameters) and the high system order $(n=7)$, the quadratic programming approach with the target simplex $\mathcal{S}$ does not give any feasible solution. So, instead of simplex $\mathcal{S}$ we used the reflection vectors polytope $\mathcal{V}(a)$ with a fixed target polynomial $e(z)=a(z)$.

The robust root locus, obtained by taking 500 uniformly distributed random plants within the uncertainty polytope, is represented in Fig. 3.

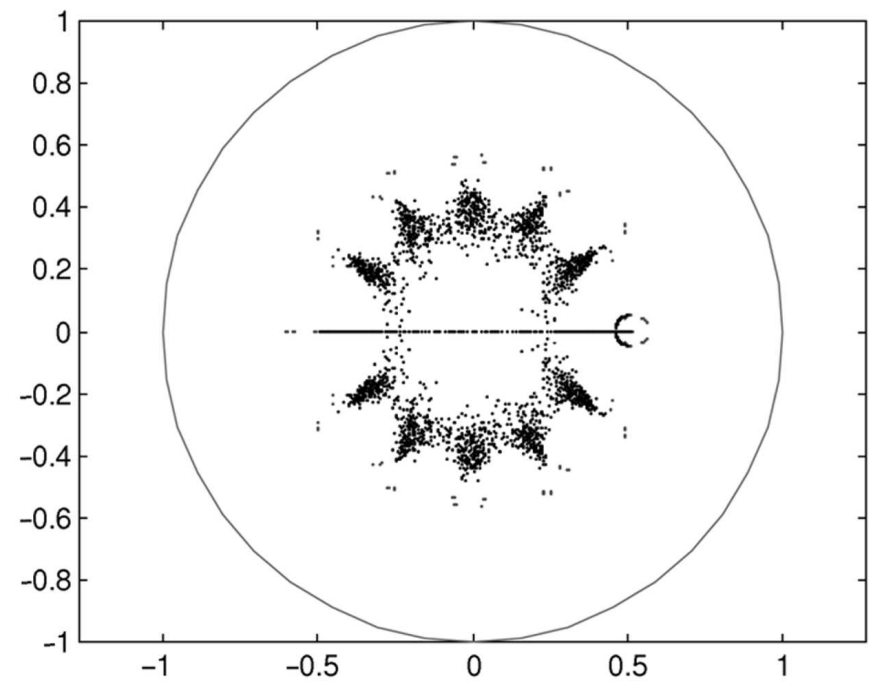

Fig. 3. Robust root locus of the robot. 
The results obtained by the 3rd-order controller are considerably better than the root locus of the system with the 7th-order controller, designed by the LMI and positive polynomial approach $\left[{ }^{9}\right]$. Indeed, for our method $\left|r_{\text {max }}\right| \approx 0.6$, whilst for LMI $\left|r_{\max }\right| \approx 0.95\left[^{9}\right]$.

\section{CONCLUSIONS}

Robust output controller design by the quadratic programming approach is based on a stability measure $\rho$, which indicates the placement of vertices of the polytope of the closed-loop system against the preselected stable target simplex.

A constructive procedure for generating stable polytopes (or simplexes) in the polynomial coefficient space is given. This procedure is quite straightforward, because it requires the choice of only one stable point (the initial polynomial), with some restrictions on its reflection coefficients. Then all the vertices of the polytope (or simplex) will be generated by reflection vectors of this point.

It is shown, first, that reflection vectors are placed on the stability boundary, with specific roots depending on the reflection vector number and the argument sign and, second, that the line segments between an arbitrary Schur polynomial and its reflection vectors are Schur stable.

\section{ACKNOWLEDGEMENT}

This work was supported by the Estonian Science Foundation (grants Nos 5405 and 6837).

\section{REFERENCES}

1. Keel, L. H. and Bhattacharyya, S. P. A linear programming approach to controller design. Automatica, 1999, 35, 1717-1724.

2. Clarke, T. and Griffin, S. J. An addendum to output feedback eigenstructure assignment: retro-assignment. Int. J. Control, 2004, 77, 78-85.

3. Duan, G. R. Parametric eigenstructure assignment via output feedback based on singular value decomposition. IEE Proc. Control Theory Appl., 2003, 150, 93-100.

4. Lordelo, A. D. S. and Ferreira, P. A. V. Interval analysis and design of robust pole assignment controllers. In Proceedings of the 41st IEEE Conference on Decision and Control, Las Vegas. IEEE Piscataway, NJ, 2002, 1461-1466.

5. Ackermann, J. Robust Control. Systems with Uncertain Physical Parameters. SpringerVerlag, London, 1993.

6. Magni, J. F. Robust Modal Control. Kluwer Academic Publishing, New York, 2002.

7. Jetto, L. Strong stabilization over polytopes. IEEE Trans. Autom. Control, 1999, 44, 12111216.

8. Rotstein, H., Sanchez Pena, R., Bandoni, J., Desages, A. and Romagnoli, J. Robust characteristic polynomial assignment. Automatica, 1991, 27, 711-715.

9. Henrion, D., Šebek, M. and Kučera, V. Positive polynomials and robust stabilization with fixed-order controllers. IEEE Trans. Autom. Control, 2003, 48, 1178-1186. 
10. Scherer, C., Gahinet, P. and Chilali, M. Multiobjective output-feedback control via LMI optimization. IEEE Trans. Autom. Control, 1997, 42, 896-911.

11. Galafiore, G. and El Ghaoui, L. Ellipsoidal bounds for uncertain linear equations and dynamical systems. Automatica, 2004, 40, 773-787.

12. Henrion, D., Peaucelle, D., Arzelier, D. and Šebek, M. Ellipsoidal approximation of the stability domain of a polynomial. IEEE Trans. Autom. Control, 2003, 48, 2255-2259.

13. Kharitonov, V. L. Asymptotic stability of a family of systems of linear differential equations. Differential Equations, 1978, 14, 1483-1485 (in Russian).

14. Chapellat, H. and Bhattacharyya, S. P. A generalization of Kharitonov's theorem: robust stability of interval plants. IEEE Trans. Autom. Control, 1989, 34, 306-311.

15. Katbab, A. and Jury, E. I. Robust Schur stability of control systems with interval plants. Int. J. Control, 1990, 51, 1343-1352.

16. Ackermann, J. and Kaesbauer, D. Stable polyhedra in parameter space. Automatica, 2003, 39, 937-943.

17. Diaz-Barrero, J. L. and Egozcue, J. J. Characterization of polynomials using reflection coefficients. Appl. Math. E-Notes, 2004, 4, 114-121.

18. Picinbono, B. and Benidir, M. Some properties of lattice autoregressive filters. IEEE Trans. Acoust. Speech Signal Process., 1986, 34, 342-349.

19. Kay, S. M. Modern Spectral Estimation. Prentice Hall, New Jersey, 1988.

20. Oppenheim, A. M. and Schaffer, R. W. Discrete-Time Signal Processing. Prentice-Hall, Englewood Cliffs, 1989.

21. Nurges, Ü. and Luus, R. Discrete Kharitonov's theorem and robust control. Control Intelligent Systems, 2002, 30, 110-118.

22. Bartlett, A. C., Hollot, C. V. and Huang, L. Root location of an entire polytope of polynomials: it suffices to check the edges. Math. Control Signals System, 1988, 1, $61-71$.

23. Nurges, Ü. New stability conditions via reflection coefficients of polynomials. IEEE Trans. Autom. Control, 2005, 50, 1354-1360.

24. Nurges, Ü. and Rüstern, E. The distance from stability boundary and reflection vectors. In Proceedings of the American Control Conference, Anchorage, USA. IEEE, Piscataway, NJ, 2002, 3908-3913.

\section{Robustne modaaljuhtimine karakteristliku polünoomi peegeldusvektorite polütoobi abil \\ Ülo Nurges}

On lahendatud robustse modaalse regulaatori sünteesi ülesanne diskreetaja süsteemidele. Kui tavalise modaalse regulaatori sünteesil tuleb valida üks stabiilne punkt suletud süsteemi karakteristliku polünoomi kordajate ruumis, siis robustsel ülesande püstitusel tuleks ette anda soovitav stabiilne simpleks või polütoop. Töös on esitatud konstruktiivne protseduur stabiilse polütoobi (simpleksi) leidmiseks sobivas stabiilsuspiirkonna osas. Esitatud protseduur on suhteliselt lihtne, sest teatud polünoomide klassi jaoks on tõestatud nende peegeldusvektorite polütoopide stabiilsus.

Robustse regulaatori süntees tugineb stabiilsusvarul, mis on defineeritud kui minimaalne kaugus valitud stabiilse simpleksi ja ebatäpse objekti (polütoopse) mudeli tippude vahel. Sünteesi ülesanne on lahendatud ruutplaneerimise meetodil. 